\title{
Etnobotânica de chás terapêuticos em Rio Urubueua de Fátima, Abaetetuba - Pará, Brasil
}

\author{
Patricia Homobono Brito de Moura ${ }^{1}$ \\ Flávia Cristina Araújo Lucas ${ }^{1 *}$ \\ Ana Cláudia Caldeira Tavares-Martinss ${ }^{1}$ \\ Gerciene de Jesus Miranda Lobato ${ }^{1}$ \\ Ely Simone Cajueiro Gurgel ${ }^{2}$ \\ ${ }^{1}$ Universidade do Estado do Pará, Centro de Ciências Naturais e Tecnologia \\ Travessa Enéas Pinheiro, 2626, CEP 66095-100, Belém - PA, Brasil \\ ${ }^{2}$ Museu Paraense Emílio Goeldi, Belém - PA, Brasil \\ * Autor para correspondência \\ copaldoc@yahoo.com.br
}

Submetido em 29/04/2015

Aceito para publicação em 12/01/2016

\section{Resumo}

Estudos etnobotânicos no Brasil revelaram os chás como principal forma de tratamento terapêutico em comunidades tradicionais. Objetivou-se valorizar os conhecimentos tradicionais na comunidade Rio Urubueua de Fátima, Abaetetuba - PA, por meio da identificação das plantas medicinais empregadas em chás e suas formas de preparo, atribuindo a estas a sua importância na terapêutica e no tratamento das enfermidades mais recorrentes, além de investigar a qualidade das águas de consumo da população, indispensável ao preparo dos chás, e a sua relação com a saúde dos ribeirinhos. A partir de entrevistas semiestruturadas realizadas com 35 interlocutores, foram calculados para as espécies: valor de importância, concordância quanto aos usos principais, concordância quanto aos usos principais corrigidos e o fator de consenso do informante. A fim de averiguar a qualidade da água, foi feita análise microbiológica de amostras coletadas em três diferentes pontos do rio Urubueua. Foram citadas 82 receitas de chás terapêuticos. Mentha sp. obteve o maior valor de importância e, juntamente com Lippia alba (Mill) N. E. Brown. e Ficus maxima Mill., o maior índice de concordância quanto ao uso principal corrigido. Os moradores na faixa etária entre 60 e 69 anos detêm vasto conhecimento de plantas e receitas a base de chás, frequentemente empregados na sua medicina tradicional. Da água utilizada pelos moradores, foram quantificados coliformes totais e termotolerantes em todas as amostras, sendo um fator de risco para a doença diarreica, mencionada em quadros clínicos recorrentes.

Palavras-chave: Amazônia; Fitoterapia; Medicina tradicional

\section{Abstract}

Ethnobotany of therapeutic teas in the Urubueua de Fátima River community in Abaetetuba, Pará, Brazil. Ethnobotanical studies in Brazil have shown that teas are the main form of therapeutic treatment in traditional communities. The aim of this research was to evaluate the knowledge of traditional practices in Urubueua de Fátima River community in Abaetetuba, Pará through identification of the plants used to prepare 
medicinal teas and the local methods of preparation. We also evaluated the importance of plants for therapy and treatment of the most frequent diseases, and investigated the quality of drinking water for the population, which is essential for the preparation of teas, and the relationship between water quality and riparian health. We conducted semi-structured interviews with 35 locals, and for each plant species used we calculated importance value, percent agreement on primary uses, percent agreement on corrected primary uses, and interviewee consensus factor. Microbiological analysis was conducted for water samples collected at three different points of Urubueua River. Eighty-two recipes of therapeutic teas were reported in total. Mentha sp. had the highest importance value, and Lippia alba (Mill) NE Brown and Ficus maxima Mill were the plants most agreed upon concerning primary reasons for use. Residents aged between 60 and 69 years hold vast knowledge of tea plants and recipes, which are often employed in traditional medicines. Total and fecal coliforms present in samples from the population water supply indicate a risk for diarrheal disease, as mentioned in recurring clinical data.

Key words: Amazonia; Phytotherapy; Traditional medicine

\section{Introdução}

Desde os tempos mais remotos a humanidade manuseia as plantas para os mais diversos fins terapêuticos, seja através de chás, cataplasmas, tinturas ou na sua forma in natura. Os chás terapêuticos são bebidas amplamente consumidads no mundo, ricas em compostos biologicamente ativos, que contribuem para a prevenção e o tratamento de várias doenças (TREVISANATO; KIM, 2000), sendo reconhecidas a partir de informações obtidas pelo seu uso tradicional e ratificadas por estudos científicos (BRASIL, 2010).

Além do valor medicinal, os chás contribuem para outros fins, como hidratação, eliminação de toxinas, controle da temperatura corporal e auxílio na digestão de alimentos (SIMÕES et al., 1995). Apesar de seus benefícios, o consumo excessivo pode ocasionar efeitos adversos (ROHMER, 2002). Amarante et al. (2011), reportando-se á infusão da folha senescente de Montrichardia linifera (Arruda) Schott, tradicionalmente preparada para o tratamento de problemas hepáticos, ressaltou que devido ao elevado teor de manganês, essa infusão é tóxica para uma ingestão superior a um litro por dia.

No Brasil, pesquisas etnobotânicas em comunidades revelaram os chás medicinais como principal tratamento de enfermidades ou alívio de sintomas (BRASILEIRO et al., 2008; SILVA; BÜNDCHEN, 2011; SILVA et al., 2012). Esses estudos consideram que os saberes de sociedades tradicionais, acumulados por gerações, contribuem efetivamente para as investigações farmacológicas e fitoquímicas, com grande economia de tempo e dinheiro (AMOROZO, 1996; 2002).
A região Amazônica, heterogênea e ambientalmente complexa, está associada a um rico perfil de matrizes culturais de suas populações tradicionais (ELISABETSKY; SETZER, 1985). Tal aspecto é aparente na Comunidade de Rio Urubueua de Fátima, Abaetetuba - PA, onde os conhecimentos sobre a aplicação das plantas medicinais confirmam a íntima relação entre os moradores e o potencial vegetal da região. Neste ambiente, que é deficientemente assistido pelo sistema de saúde, os chás destacam-se como a principal forma de tratamento para diversas doenças.

Esta pesquisa teve por objetivo valorizar os conhecimentos tradicionais na comunidade Rio Urubueua de Fátima, Abaetetuba - PA, através da identificação das plantas medicinais empregadas em chás e suas formas de preparo, atribuindo a estas a sua importância na terapêutica e no tratamento das enfermidades mais recorrentes, além de investigar a qualidade das águas de consumo da população, indispensável ao preparo dos chás, e a sua relação com a saúde dos ribeirinhos.

\section{Material e Métodos}

\section{Área de estudo}

A comunidade Rio Urubueua de Fátima (01³7'92"S; 4858’42”W) situa-se no município de Abaetetuba - PA, pertence à mesorregião do nordeste paraense e faz parte de um conjunto de 72 ilhas localizadas entre o rio Pará e a Baía do rio Capim (0142’008”'S; 4854’009”W) (Figura 1). O clima 
FIGURA 1: Mapa de localização da comunidade Rio Urubueua de Fátima, Abaetetuba - PA, Brasil.

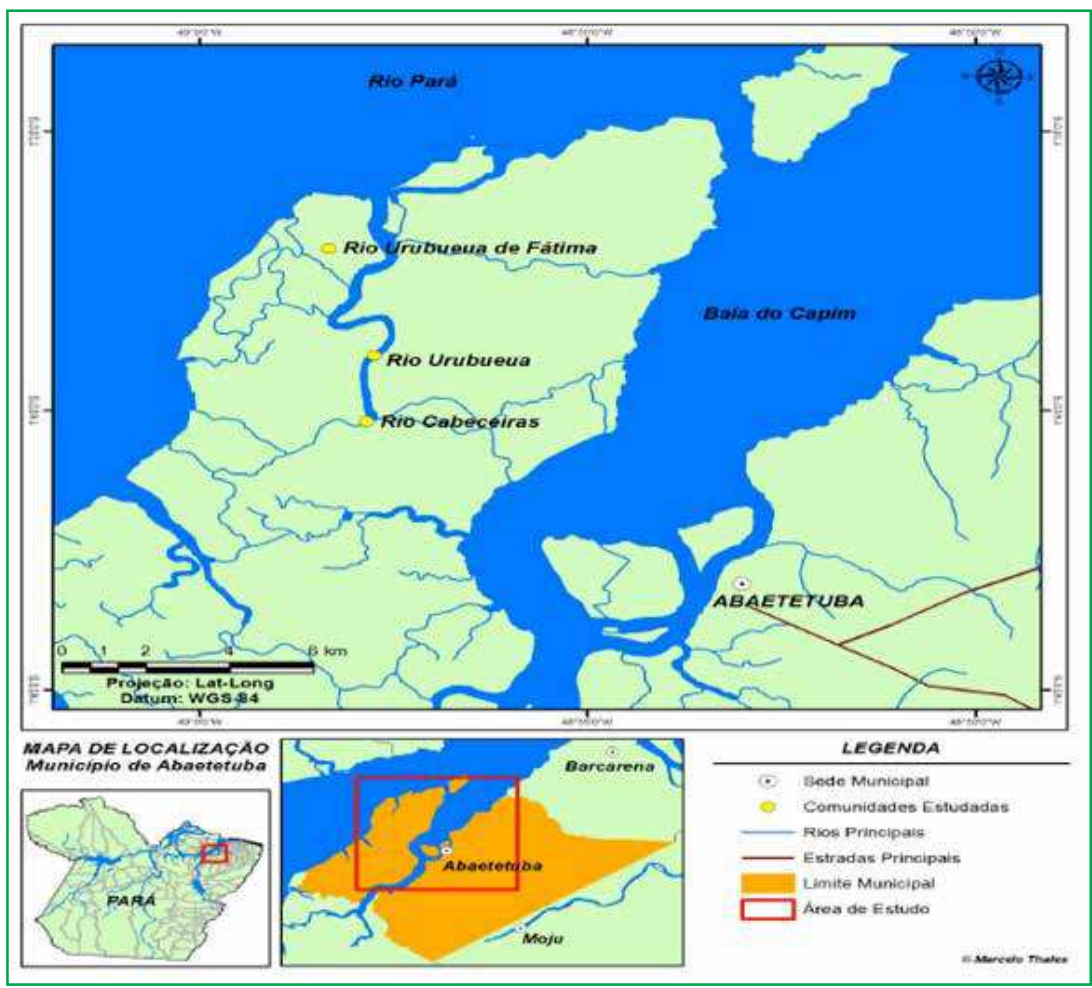

da região é super úmido, com altas temperaturas e precipitações constantes.

\section{Os ribeirinhos de Rio Urubueua de Fátima}

Na comunidade Rio Urubueua de Fátima, as unidades habitacionais, em geral, estão situadas às margens do rio ou dos furos de rios, construídas em madeira e suspensas devido ao movimento das marés. Grande parte possui um pequeno trapiche que auxilia no embarque e desembarque de pessoas e mercadorias, caracterizando o típico cenário ribeirinho do estuário amazônico. O acesso entre as moradias é feito por embarcações, e as que estão mais próximas, por estivas (pontes), as quais são geralmente feitas com o "miriti" (Mauritia flexuosa L. F.).

Dos 35 interlocutores, 32 citaram que o manejo de "açaí" (Euterpe oleracea Mart.) é a principal fonte de renda durante o segundo semestre do ano (setembro a dezembro). O palmito é extraído apenas quando são derrubadas as palmeiras mais altas para fazer o manejo das touceiras. A atividade da pesca, geralmente praticada por homens, e o extrativismo vegetal complementam a renda dos moradores durante os primeiros meses do ano.

\section{Situação de saúde na comunidade}

Em Rio Urubueua de Fátima há um Posto de Saúde, de responsabilidade da Prefeitura de Abaetetuba. No posto faltam medicamentos e a atenção dispensada à saúde dos ribeirinhos é insuficiente. Para os tratamentos mais específicos, os moradores se deslocam até o centro do município ou para Belém. Apenas uma técnica em enfermagem, responsável pelo posto, realiza os atendimentos de urgência e emergência.

As doenças que mais acometem os moradores são hipertensão, diarreias, vermes, gripes, dores e ferradas de arraia. A ocorrência de diarreia e parasitoses intestinais pode ser atribuídas à falta de saneamento básico, pois na comunidade não há tratamento de água e esgoto. A água consumida é desinfetada pela própria população com solução de hipoclorito de sódio distribuído pelos Agentes Comunitários de Saúde para as famílias cadastradas na 
Secretaria de Saúde do Município, porém nem todas possuem tal registro e por isso não recebem o produto regularmente.

\section{A seleção da comunidade e dos informantes}

Rio Urubueua de Fátima foi selecionada a partir de visitas exploratórias que evidenciaram a importância do uso das plantas medicinais, sobretudo como chá, para o tratamento de doenças ou para alívio sintomático.

Os objetivos do estudo foram apresentados em reunião aos moradores juntamente com o líder comunitário e, com a concordância das condições da pesquisa pelos ribeirinhos, foi assinado o Termo de Anuência Prévia - TAP. Posteriormente, a pesquisa foi autorizada pelo Conselho de Gestão do Patrimônio Genético (CGEN) sob o número de registro no 148/2013.

A amostra populacional deste trabalho foi de 35 interlocutores, dos gêneros masculino $(5,7 \%)$ e feminino (94,3\%), com idade entre 28 e 93 anos. A faixa etária de 50 a 59 anos representou o maior número de população amostral $(n=12)$. A escolha dos interlocutores foi por amostragem não probabilística, por meio do método bola de neve (BAILEY, 1982), em que um informante indica uma pessoa que ele julga ser conhecedora do uso de plantas, além do auxílio de um interlocutor principal que indicava pessoas que detinham conhecimento sobre as plantas medicinais. Foram também identificados os especialistas locais (ALBUQUERQUE et al., 2010), que são os moradores identificados pela população como profundos conhecedores de plantas medicinais e dotados de experiências tanto na formulação das receitas quanto nos cultivos e propagação.

\section{Coleta e análise de dados}

Foram realizadas três viagens no período de julho de 2011 a julho de 2012, cada uma com duração de uma semana. As técnicas para a coleta de dados envolveram observação participante, entrevistas semiestruturadas, listagem livre e indução não específica (ALBUQUERQUE et al., 2010). As entrevistas semiestruturadas buscaram informações sociodemográficas, etnobotânicas de plantas medicinais e etnofarmacológicas (doenças mais comuns). Após as entrevistas, quando o informante se disponibilizava, ocorriam turnês guiadas (ALBUQUERQUE et al., 2010), que consistiam em caminhadas ao terreno (área de mata mais distante da casa) ou ao quintal (situado nos arredores da casa), acompanhadas dos entrevistados para a identificação e coleta de plantas.

As amostras botânicas coletadas seguiram Martinsda-Silva (2000) e foram incorporadas no herbário (MG) do Museu Paraense Emílio Goeldi. Os nomes científicos e autorias foram atualizados de acordo com as bases online da Lista de Espécies da Flora do Brasil (http:// floradobrasil.jbrj.gov.br/), e, quando necessário, também foi consultado o banco de dados do Missouri Botanical Garden (MOBOT).

A fim de analisar a proporção de informantes que citaram uma espécie como a mais importante, calculouse o Valor de Importância $\left(\mathrm{IV}_{\mathrm{s}}\right)$, metodologia adaptada por Silva et al. (2010), com a seguinte fórmula:

$$
I V_{s}=\frac{n_{i s}}{n}
$$

em que:

$n_{i s}=$ número de informantes que consideram a espécie s mais importante; $n=$ total de informantes.

Calculou-se também a importância relativa das plantas na comunidade quanto ao número de informantes e à concordância dos usos citados. Para isso, utilizouse o método adaptado por Amorozo e Gély (1988), em que a porcentagem de concordância quanto aos usos principais (CUP) é:

$$
C U P=\frac{n^{o} \text { de informantes que citaram usos principais } \times 100}{n^{o} \text { de informantes que referiram uso da espécie }}
$$

O valor de CUP encontrado foi multiplicado por um fator de correção (CUPc), que corresponde ao número de informantes que mencionaram a espécie, dividido pelo número de informantes que mencionaram a espécie mais citada, no caso: 


$$
C U P_{C}=C U P \times F C
$$

$$
F C=\frac{n^{\circ} \text { de informantes que citaram a espécie }}{n^{\underline{o}} \text { de informantes que referiram a espécie mais citada }}
$$

Para analisar os sistemas corporais (categorias de doenças ou sintomas) com maior importância relativa dentro da comunidade, utilizou-se o Fator de Consenso do Informante (FCI) (TROTTER; LOGAN, 1986), com base na Classificação Estatística Internacional de Doenças e Problemas Relacionados à Saúde (CID-10) (OMS, 2008), com adaptações, inserindo-se a categoria "doenças culturais". Obteve-se o FCI por meio da fórmula:

$$
F C I=\frac{n_{u r}-n_{t}}{n_{u r}-1}
$$

em que:

$F C I=$ fator de consenso do informante; $n_{u r}=$ número de citações de uso em cada categoria de doença; $n_{t}=$ número de espécies usadas nessa categoria.

A CUP, a CUPc e o FCI foram calculados a partir das plantas indicadas para tratamento com os chás. Os chás foram classificados em duas classes: simples, os que são preparados com uma espécie, e mistos, quando são utilizadas mais de uma (BRASIL, 1998).

\section{Análise microbiológica da água}

A análise microbiológica da água foi feita pelo Las Sanches Laboratório, que segue os métodos presentes no Standard Methods for The Analysis Of Water And Wastewater 20th por meio da metodologia dos tubos múltiplos, que avalia a presença ou ausência de coliformes totais e termotolerantes por número mais provável por $100 \mathrm{~mL}$ de amostra (NMP/100 mL).

Dentro do grupo total de moradias, buscou-se aleatoriamente três residências para a coleta de água. Em cada uma coletaram-se duas amostras, uma proveniente do rio, e a outra, do pote de cerâmica que armazena a água tratada pelos moradores. A água é considerada tratada quando desinfetada com hipoclorito de sódio ou fervida. Ao todo seis amostras de água foram coletadas e analisadas.

\section{Resultados}

\section{Usos e potencialidades das plantas medicinais}

Todos os chás indicados foram descritos pelos interlocutores para prevenir, tratar e curar enfermidades. Assim, as plantas medicinais preparadas como chá representam o primeiro recurso de tratamento para as doenças ou sintomas adquiridos. Os 35 interlocutores listaram 82 receitas de chás, que incluem 58 espécies (Tabela 1).

Foram listadas 34 famílias botânicas, com maior número de espécies Fabaceae (7) e Lamiaceae (6), sendo as herbáceas as mais citadas (48\%). Na comunidade, as ervas são comumente mantidas em paneiros, latas ou baldes, que ficam suspensos em uma estrutura denominada jirau, devido à dificuldade de cultivo em solos de várzea.

Dos chás terapêuticos, $52,4 \%$ são preparados com apenas uma espécie (chás simples) e 47,6\% com mais de uma espécie (chás mistos), sendo a folha a parte mais empregada (64,8\%), seguida da casca (16,5\%). Do total, 12 chás tiveram três ou mais menções e representaram 46,6\% das citações. O de Lippia alba foi citado por 12 interlocutores, principalmente como anti-hipertensivo e calmante, sendo também recomendado contra dores de estômago e febres persistentes: 'Toma o chá de cidreira fervido bem quente, e é logo que a pessoa sua e passa aquela febre' (Entrevista com F. D. T., 63 anos, em $28 / 05 / 12$ ).

O número de chás listados foi superior entre os interlocutores que se encontram na faixa etária de 60 a 69 anos, ocorrendo poucos relatos entre os de 80 a 99 anos, pois neste grupo foi revelado que muitas receitas já tinham sido esquecidas. 
TABELA 1: Espécies empregadas nos chás terapêuticos. Av: Arbóreo. Ab: Arbustivo. E: Erva. C: Cultivada. Es: Espontânea.

\begin{tabular}{|c|c|c|c|c|}
\hline Etnoespécie & Espécie & Família & Hábito & Origem \\
\hline Abacate & Persea americana Mill. & Lauraceae & $\mathrm{Av}$ & $\mathrm{C}$ \\
\hline Açaí & Euterpe oleracea Mart. & Arecaceae & Av & $\mathrm{Es} / \mathrm{C}$ \\
\hline Alho & Allium sativum $\mathrm{L}$. & Amaryllidaceae & $\mathrm{E}$ & $\mathrm{C}$ \\
\hline Amor crescido & Portulaca pilosa $\mathrm{L}$. & Portulacaceae & $\mathrm{E}$ & $\mathrm{C}$ \\
\hline Anoerá & Licania laxiflora Fritsch & Chrysobalanaceae & Av & Es \\
\hline Arruda & Ruta graveolens $\mathrm{L}$. & Rutaceae & $\mathrm{E}$ & $\mathrm{C}$ \\
\hline Aturiá & Machaerium lunatum (L. f.) Ducke & Fabaceae & $\mathrm{Ab}$ & Es \\
\hline Babosa & Aloe vera $\mathrm{L}$. & Xanthorrhoeaceae & $\mathrm{E}$ & $\mathrm{C}$ \\
\hline Banana Roxa & Musa paradisiaca $\mathrm{L}$ & Musaceae & $\mathrm{E}$ & $\mathrm{C}$ \\
\hline Barbatimão & Stryphnodendron barbatimam Mart. & Fabaceae & $\mathrm{Ab}$ & $\mathrm{C}$ \\
\hline Boldo & Vernonia condensata Baker & Asteraceae & $\mathrm{E}$ & $\mathrm{C}$ \\
\hline Caju & Anacardium occidentale L. & Anacardiaceae & Av & $\mathrm{C}$ \\
\hline Canaficha & Costus lasius Loes. & Costaceae & $\mathrm{E}$ & $\mathrm{C}$ \\
\hline Canela & Cinnamomum zeylanicum Blume & Lauraceae & $\mathrm{Ab}$ & $\mathrm{C}$ \\
\hline Capim marinho & Cymbopogon citratus (DC.) Stapf & Poaceae & $\mathrm{E}$ & $\mathrm{C}$ \\
\hline Castanha do Pará & Bertholletia excelsa Bonpl. & Lecythidaceae & Av & Es \\
\hline Catinga de mulata & Aeollanthus suaveolens Mart. ex Spreng. & Lamiaceae & $\mathrm{E}$ & $\mathrm{C}$ \\
\hline Caxinguba & Ficus maxima Mill. & Moraceae & Av & Es \\
\hline Chicória & Eryngium foetidum $\mathrm{L}$. & Apiaceae & $\mathrm{E}$ & $\mathrm{C}$ \\
\hline Cidreira & Lippia alba (Mill) N. E. Brown. & Verbenaceae & $\mathrm{E}$ & $\mathrm{C}$ \\
\hline Comida de jabuti & Peperomia pellucida (L.) Kunth & Piperaceae & $\mathrm{E}$ & Es \\
\hline Coramina & Pedilanthus tithymaloides Poit. & Euphorbiaceae & $\mathrm{E}$ & $\mathrm{C}$ \\
\hline Corrente & Pfaffia glomerata (Spreng.) Pedersen & Amaranthaceae & $\mathrm{E}$ & $\mathrm{C}$ \\
\hline Embaúba & Cecropia distachya Huber & Urticaceae & Av & $\mathrm{E}$ \\
\hline Escada de jabuti & Bauhinia guianensis Aubl. & Fabaceae & Av & $\mathrm{E}$ \\
\hline Gengibre & Zingiber officinale Roscoe & Zingiberaceae & $\mathrm{E}$ & $\mathrm{C}$ \\
\hline Goiaba & Psidium guajava $\mathrm{L}$. & Myrtaceae & Av & $\mathrm{C} / \mathrm{Es}$ \\
\hline Hortelã & Mentha sp. & Lamiaceae & $\mathrm{E}$ & $\mathrm{C}$ \\
\hline Jatobá & Hymenaea courbaril $\mathrm{L}$. & Fabaceae & Av & Es \\
\hline Jucá & Libidibia ferrea (Mart. ex. Tul.) L.P.Queiroz & Fabaceae & Av & $\mathrm{C}$ \\
\hline Limão & Citrus $\times$ limonia $($ L.) Osbeck & Rutaceae & $\mathrm{Ab}$ & $\mathrm{C}$ \\
\hline Lombrigueira & Spigelia hamelioides Kunth & Loganiaceae & $\mathrm{E}$ & Es \\
\hline Malvarisco & Piper marginatum Jacq. & Piperaceae & $\mathrm{E}$ & $\mathrm{C}$ \\
\hline Mamão macho & Carica papaya $\mathrm{L}$ & Caricaceae & Av & $\mathrm{C}$ \\
\hline Mangueiro & Rhizophora racemosa G.Mey. & Rhizophoraceae & Av & Es \\
\hline Manjerona salva & Lippia thymoides Mart. \& Schauer & Verbenaceae & $\mathrm{E}$ & $\mathrm{C}$ \\
\hline Marcela / Macela & Pluchea sagittalis (Lam.) Cabrera & Asteraceae & $\mathrm{E}$ & Es \\
\hline Marupazinho & Eleutherine plicata (Sw.) Herb. & Iridaceae & $\mathrm{E}$ & $\mathrm{C}$ \\
\hline Mastruz & Chenopodium ambrosioides L. & Amaranthaceae & $\mathrm{E}$ & $\mathrm{C}$ \\
\hline Meracelina & Alternanthera dentata (Moench) Stuchlík ex R.E.Fr. & Amaranthaceae & $\mathrm{Ab}$ & $\mathrm{C}$ \\
\hline Óleo elétrico de planta & Piper callosum Ruiz \& Pav. & Piperaceae & $\mathrm{E}$ & $\mathrm{C}$ \\
\hline Oriza & Pogostemon heyneanus Benth. & Lamiaceae & $\mathrm{E}$ & $\mathrm{C}$ \\
\hline Pariri & Fridericia chica (Bonpl.) L.G.Lohmann & Bignoniaceae & $\mathrm{E}$ & $\mathrm{C}$ \\
\hline Pata de vaca & Bauhinia forficata Link & Fabaceae & Av & $\mathrm{C}$ \\
\hline Pirarucu & Kalanchoe pinnata (Lam.) Pers. & Crassulaceae & $\mathrm{E}$ & $\mathrm{C}$ \\
\hline Quebra-pedra & Phyllanthus niruri $\mathrm{L}$. & Phyllanthaceae & $\mathrm{E}$ & $\mathrm{Es} / \mathrm{C}$ \\
\hline Sabugueiro & Sambucus nigra L. & Adoxaceae & $\mathrm{Ab}$ & $\mathrm{C}$ \\
\hline Sacaca & Croton cajucara Benth. & Euphorbiaceae & Av & $\mathrm{C}$ \\
\hline Salva do Marajó & Hyptis crenata Pohl ex Benth. & Lamiaceae & $\mathrm{E}$ & $\mathrm{C}$ \\
\hline Sete dores & Coleus sp. & Lamiaceae & $\mathrm{E}$ & $\mathrm{C}$ \\
\hline Sucuba & Himatanthus sucuuba (Spruce ex Müll.Arg.) Woodson & Apocynaceae & Av & Es \\
\hline Sucuriju & Mikania lindleyana DC. & Asteraceae & $\mathrm{L}$ & $\mathrm{C}$ \\
\hline Tansagem & Plantago major L. & Plantaginaceae & $\mathrm{E}$ & $\mathrm{C}$ \\
\hline Urtiga mansa & Coleus amboinicus (Lour.) Spreng. & Lamiaceae & $\mathrm{E}$ & $\mathrm{C}$ \\
\hline Urubucaá & Aristolochia trilobata L. & Aristolochiaceae & $\mathrm{E}$ & $\mathrm{C}$ \\
\hline Vassourinha de botão & Scoparia dulcis L. & Plantaginaceae & $\mathrm{E}$ & Es \\
\hline Verônica & Dalbergia monetaria L. f. & Fabaceae & $\mathrm{L}$ & Es \\
\hline Vinagreira & Hibiscus sabdariffa L. & Malvaceae & $\mathrm{Ab}$ & $\mathrm{C}$ \\
\hline
\end{tabular}




\section{Valor de importância das plantas medicinais}

Este valor de importância foi calculado com base nas plantas mais importantes para a população, que totalizaram 18 espécies. Mentha sp. $(0,17)$, indicada principalmente contra diarreias, dores de barriga e vermes, e Vernonia condensata $(0,14)$, para o tratamento de dores do estômago, são as que tiveram o maior índice, estando ambas direcionadas ao transtorno do sistema gastrintestinal.

\section{Importância relativa das espécies}

A percentagem de concordância quanto aos usos principais (CUP), fator de correção e a CUP corrigida das plantas com quatro ou mais citações encontra-se na Tabela 2.
Portulaca pilosa, Eleutherine plicata, Libidibia ferrea e Croton cajucara mostraram o valor máximo de CUP, porém o maior valor de CUPc foi atribuído a Mentha sp., Lippia alba e Ficus maxima, com índices de $61,1 \%$. Baixo índice de consenso de uso pode indicar a versatilidade de uma planta, que pode ser usada para vários tratamentos.

\section{Fator de Consenso dos Informantes (FCI)}

Os resultados sobre o Fator de Consenso dos Informantes são apresentados na Tabela 3.

TABELA 2: CUP e CUPc das espécies com quatro ou mais citações. Fc corresponde ao Fator de correção.

\begin{tabular}{lclcccc}
\hline Etnoespécie & $\begin{array}{c}\text { Informantes que } \\
\text { citaram o uso da } \\
\text { espécie }\end{array}$ & \multicolumn{1}{c}{ Usos principais } & $\begin{array}{c}\text { Informantes que } \\
\text { citaram usos } \\
\text { principais }\end{array}$ & CUP & Fc & $\begin{array}{c}\text { CUPc } \\
\text { (\%) }\end{array}$ \\
\hline Açaí & 9 & Diarreia & 8 & 88,9 & 0,5 & 44,4 \\
Amor crescido & 5 & Dor no estômago & 5 & 100,0 & 0,3 & 27,8 \\
Anoerá & 5 & Vermes & 3 & 60,0 & 0,3 & 16,7 \\
Boldo & 10 & Dor de estômago & 8 & 80,0 & 0,6 & 44,4 \\
Caxinguba & 13 & Vermes & 11 & 84,6 & 0,7 & 61,1 \\
Cidreira & 12 & Hipertensão e calmante & 11 & 91,7 & 0,7 & 61,1 \\
Goiaba & 10 & Diarreia & 9 & 90,0 & 0,6 & 50,0 \\
Hortelã & 18 & Diarreia, vermes & 11 & 61,1 & 1,0 & 61,1 \\
Jucá & 6 & Infecção, cicatrizante, gastrite & 6 & 100,0 & 0,3 & 33,3 \\
Marupazinho & 6 & Dor de barriga, diarreia & 6 & 100,0 & 0,3 & 33,3 \\
Mastruz & 6 & Vermes, gastrite & 5 & 83,3 & 0,3 & 27,8 \\
Óleo elétrico & 5 & Dor de barriga & 2 & 40,0 & 0,3 & 11,1 \\
Pariri & 8 & Anemia & 8 & 100,0 & 0,4 & 44,4 \\
Quebra-pedra & 4 & Pedra nos rins & 2 & 50,0 & 0,2 & 11,1 \\
Sacaca & 4 & Dor no estômago & 4 & 100,0 & 0,2 & 22,2 \\
Sucuba & 9 & Pneumonia, tosse & 8 & 88,9 & 0,5 & 44,4 \\
Sucuriju & 7 & Dor no estômago & 6 & 85,7 & 0,4 & 33,3 \\
Verônica & 8 & Anemia, dor de urina & 6 & 75,0 & 0,4 & 33,3 \\
\hline
\end{tabular}


O sistema corporal de maior concordância quanto ao uso das espécies foi o relacionado às doenças do sangue e dos órgãos hematopoiéticos, com FCI de 83\%, e dentro desta categoria destaca-se o "pariri", citado por oito interlocutores. As "doenças culturais" designadas pela comunidade como quebranto e dentição obtiveram $71 \%$ de consenso entre os informantes.

Os transtornos do sistema circulatório tiveram indicação de oito espécies, com destaque a Lippia alba, usada como anti-hipertensiva e 12 vezes mencionada. Para as doenças respiratórias, a Himatanthus sucuuba foi citada 11 vezes, e quanto aos transtornos do sistema digestivo, três espécies são repetidamente ressaltadas, Vernonia condensata (10), Psidium guajava (11) e Mentha sp. (12).

Nas doenças do aparelho geniturinário, a D. monetaria obteve $80 \%$ das citações para o tratamento da infecção urinária, descrita como "dor de urina".

\section{Qualidade da água}

Em todas as amostras de água in natura foram quantificados índices de coliformes termotolerantes (Tabela 4).

TABELA 3: Fator de consenso dos informantes entre os sistemas corporais e o número de espécies relacionadas.

\begin{tabular}{lcccc}
\hline \multicolumn{1}{c}{ Sistemas Corporais } & Espécies & \% espécies & Citações de uso & FCI (\%) \\
\hline Doenças infecciosas e parasitárias & 15 & 26,32 & 36 & 60 \\
Doenças das glândulas endócrinas, da nutrição e do & 5 & 8,77 & 9 & 50 \\
metabolismo & 3 & 5,26 & 13 & 83 \\
Doenças do sangue e dos órgãos hematopoiéticos & 3 & 5,26 & 5 & 50 \\
Transtornos do sistema nervoso & 8 & 14,04 & 22 & 67 \\
Transtornos do sistema circulatório & 10 & 17,54 & 27 & 65 \\
Transtornos do sistema respiratório & 35 & 61,40 & 110 & 69 \\
Transtornos do sistema digestivo & 6 & 10,53 & 13 & 58 \\
Transtornos do sistema geniturinário & 2 & 3,51 & 4 & 67 \\
Doenças da pele e do tecido celular subcutâneo & 3 & 5,26 & 8 & 71 \\
"Doenças culturais" & & &
\end{tabular}

TABELA 4: Valores de referência e quantificação de coliformes totais e termotolerantes nas amostras de água coletadas das moradias em NMP/100 mL. C. To: Coliformes totais. C. Tt.: Coliformes termotolerantes. VR: Valor de referência. I: Moradia 1. II: Moradia 2. III: Moradia 3.

\begin{tabular}{|c|c|c|c|c|c|c|c|c|}
\hline \multirow{2}{*}{ Grupo } & \multirow{2}{*}{ VR para consumo ${ }^{1}$} & \multirow{2}{*}{ VR para balneabilidade ${ }^{2}$} & \multicolumn{3}{|c|}{ Água do rio } & \multicolumn{3}{|c|}{ Água tratada } \\
\hline & & & I & II & III & I & II & III \\
\hline C. To. & Tolerada $^{3}$ & Não estabelecido & 11.000 & 360 & 4.600 & 0 & 11.000 & 0 \\
\hline C. Tt. & Ausência & $\begin{array}{c}\text { Excelente } \leq 250 \\
\text { Muito Boa } \leq 500 \\
\text { Satisfatória } \leq 1.000 \\
\text { Imprópria } \geq 2.500\end{array}$ & 230 & 36 & 2.300 & 0 & 36 & 0 \\
\hline
\end{tabular}

Valores de referência do Ministério da Saúde (BRASIL, 2000b); ${ }^{2}$ Valores de Referência do Conselho Nacional do Meio Ambiente (BRASIL, 2000a); ${ }^{3}$ Em locais onde não há rede de distribuição de água. 
No ponto 2, foi detectado o grupo dos termotolerantes na amostra tratada, consequentemente imprópria para consumo humano. Esse fato aponta para desinfecção inadequada, com concentração abaixo do recomendado de hipoclorito de sódio ou até mesmo ausência deste, já que a distribuição do produto é insuficiente para toda a população da comunidade. Outro agravante a ser considerado é a higienização instisfatória dos vasilhames que acondicionam a água, o que pode ter sido fator preponderante no elevado índice de bactérias presente.

\section{Discussão}

\section{A comunidade Rio Urubueua de Fátima e os chás medicinais}

O modo de vida destes ribeirinhos se caracteriza pelo uso expressivo de plantas medicinais como primeiro recurso de tratamento, e os interlocutores entre 60 e 69 anos são detentores de maior conhecimento sobre os chás e das espécies vegetais cultivadas nos quintais. Segundo Silva e Bündchen (2011), a obtenção das plantas medicinais a partir de cultivos caseiros é essencial para a preservação e transmissão do conhecimento etnobotânico.

As mulheres são as principais mantenedoras desse conhecimento, e comumente o papel feminino nas farmacopeias populares é majoritário e correlacionado ao fato de elas serem as responsáveis pela atenção básica à saúde da família (FERRÃO et al., 2014), característica bastante comum nos costumes da comunidade, onde os homens são responsáveis pelas atividades com o manejo do açaí e a pesca, e as mulheres dedicam-se à atenção familiar.

A folha é a parte mais empregada para o preparo dos chás medicinais. Para Silva et al. (2012) o maior uso das folhas pode ser associado à facilidade de coletá-las, uma vez que estas se fazem presentes por todo ano, e à conservação da planta, pois sua retirada não prejudica o vegetal (MARODIN; BAPTISTA, 2002).

As espécies citadas em Rio Urubueua pelos moradores também foram reportadas nas pesquisas de Martins et al. (2005), na Ilha do Combu, Belém,
PA, com 20 espécies comuns, e destas, 12 com as mesmas indicações. Em Amorozo e Gély (1988), nas comunidades Vila de Itupanema e Vila Nova do Piry em Barcarena, PA, notou-se o uso comum de 42 plantas, com 20 delas empregadas para as mesmas doenças. Nos estudos de Pinto e Barbosa (2009), em Igarapé Miri, PA, há uso semelhante de 55 plantas, e de 42 para tratamentos similares.

A concordância de uso das plantas com fins semelhantes compreende uma informação etnodirigida de suma importância em pesquisas farmacológicas, uma vez que muitas plantas utilizadas por diferentes comunidades tiveram seu princípio ativo comprovado (AMOROZO; GÉLY, 1988). Algumas das mais conhecidas substâncias bioativas, como a atropina, a efedrina e a reserpina, foram introduzidas após estudos desenvolvidos a partir da medicina popular, isto é, a seleção etnofarmacológica favorece, com maior probabilidade, a descoberta de novas substâncias ativas (MACIEL et al., 2002).

A concordância de uso principal corrigida aponta as utilizações mais populares e aceitas para uma espécie entre os interlocutores, o que, segundo Vendruscolo e Mentz (2006), pode evidenciar mais segurança quanto à validade das indicações. Dessa forma, a CUPc aponta quais espécies devem ter prioridade para estudos de bioprospecção (THRING; WEITZ, 2006).

As plantas com índices de CUPc maiores que 50\% têm significativa concordância de usos, fator que pode indicar potencial fitoterápico, funcionando como uma pré-triagem dentro dos estudos etnofarmacológicos (SILVA; PROENÇA, 2008).

O chá de Lippia alba foi o mais citado, e Mentha sp. obteve maior IVs e CUPc, mostrando a importância dessas espécies para a comunidade. Outro fato que representa essa valorização é que as mesmas plantas são indicadas para o tratamento das categorias de doenças mais comuns relatadas pelos moradores, como hipertensão (Lippia alba) e diarreias (Mentha sp.), portanto se mostram com versatilidade de uso.

Muitas espécies listadas na comunidade se referem ao tratamento dos transtornos do sistema digestivo, tendência encontrada em outros estudos realizados 
no Brasil (AMOROZO, 2002; PILLA et al., 2006; NEGRELLE; FORNAZZARI, 2007; BALDAUF et al., 2009), seguido das doenças infecciosas e parasitárias. Entretanto, os chás medicinais em Rio Urubueua de Fátima não tratam apenas as doenças descritas pela Organização Mundial da Saúde, mas também as enfermidades estabelecidas dentro da cultura da comunidade, como o quebranto e a dentição.

A importância das plantas não nativas na terapêutica local é um forte traço identitário em Rio Urubueua de Fátima. Para Bennett e Prance (2000), a utilização de plantas exóticas na farmacopeia vegetal de povos indígenas e mestiços do Norte da América do Sul pode ser um reflexo da herança cultural europeia na colonização da Amazônia. O rio Amazonas serviu de rota na conquista da região pelos portugueses e espanhóis, sendo a várzea a primeira fronteira de expansão da colonização (PORRO, 1995), logo, o habitante atual da várzea é herdeiro das culturas indígenas e europeias, e essa interação pode estar ligada ao uso de plantas não nativas da região.

\section{Qualidade da água}

A falta de saneamento básico na comunidade e a proximidade dos rios é um fator de risco para o aumento do número de coliformes termotolerantes na rede hidrográfica. Em locais onde não há distribuição de água canalizada, o Ministério da Saúde por meio da portaria 1.469/2001 (BRASIL, 2000b) permite a presença de coliformes totais na água para consumo humano, desde que na ausência dos termotolerantes e/ou E. coli.

A higiene corporal dos moradores é feita diretamente nos rios e faz parte da cultura popular. A balneabilidade das águas, definida pelo Conselho Nacional do Meio Ambiente, pela resolução 274 de 2000 (BRASIL, 2000a), é apropriada, pois possuem concentração de coliformes termotolerantes menores que $2.500 \mathrm{NMP} / 100$ $\mathrm{mL}$. Os pontos 1 e 2 podem ser considerados excelentes, segundo a resolução citada, pois possuem concentração menor que $250 \mathrm{NMP} / 100 \mathrm{~mL}$, porém no ponto 3 é possível notar a proximidade da concentração máxima permitida. Estudos realizados por Alves et al. (2012) no Rio Ararí, arquipélago do Marajó - PA, geograficamente próximo ao rio Urubueua, indicaram níveis similares a este trabalho quanto à concentração de coliformes termotolerantes, de 350 a $2.400 \mathrm{NMP} / 100 \mathrm{~mL}$.

O modo de uso mais reportado dos chás é na forma de decocção, e de acordo com os parâmetros de qualidade da água essa preparação é a mais adequada, pois a alta temperatura combate os coliformes termotolerantes. Porém, a detecção destes micro-organismos patogênicos em todos os pontos do rio aponta a necessidade de tratamento da água para o consumo da população, pois a presença destas bactérias é indicadora de risco para a doença diarreica, citada como uma das mais frequentes enfermidades na área de estudo.

$\mathrm{Na}$ comunidade Rio Urubueua de Fátima, o conhecimento sobre o potencial vegetal compõe um importante traço cultural para os ribeirinhos, em que o saber sobre as plantas medicinais é geralmente repassado de forma oral. As plantas utilizadas para o preparo dos chás englobam tanto espécies nativas quanto exóticas, demonstrando que a flora local não é a única fonte para o tratamento médico na região. $\mathrm{O}$ grande número de espécies relatadas na terapêutica de transtornos do sistema digestivo, pricipalmente as diarreias, demonstra a importância dessa classe de doenças e a necessidade real de um tratamento hídrico eficiente.

\section{Referências}

ALBUQUERQUE, U. P.; LUCENA, R. F. P; CUNHA, L. V. F. C. Métodos e técnicas na pesquisa etnobiológica e etnoecológica. (Coleção Estudos e Avanços). NUPPEA: Recife, 2010. 559 p.

ALVES, I. C. C.; EL-ROBRINI, M.; SANTOS, M. L. S.; MONTEIRO, S. M.; BARBOSA, L. P. F.; GUIMARÃES, J. T. F. Qualidade das águas superficiais e avaliação do estado trófico do Rio Arari (Ilha de Marajó, norte do Brasil). Acta Amazonica, Manaus, v. 42, n. 1, p. 115-124, 2012

AMARANTE, C. B.; SILVA, J. C. F.; MÜLLER, R. C. S. Avaliação da composição mineral do chá da folha senescente de Montrichardia linifera (Arruda) Schott (Araceae) por espectrometria de absorção atômica com chama (FAAS). Química Nova, São Paulo, v. 34, n. 3, p. 419-423, 2011.

AMOROZO, M. C. M. A abordagem etnobotânica na pesquisa de plantas medicinais. In: DI STASI, L. C. (Org.). Plantas medicinais: arte e ciência - Um guia de estudo interdisciplinar. São Paulo: Editora da Universidade Estadual Paulista, 1996. 230 p.

AMOROZO, M. C. M. Uso e diversidade de plantas medicinais em Santo Antonio do Leverger, MT, Brasil. Acta Botanica Brasilica, Feira de Santana, v. 16, n. 2, p. 189-203, 2002. 
AMOROZO, M. C. M.; GÉLY, A. L. Uso de plantas medicinais por caboclos do Baixo Amazonas. Boletim do Museu Paraense Emilio Goeldi, Belém, v. 4, n. 1, p. 47-131, 1988.

BAILEY, K. D. Methods of social research. New York: The Free Press, 1982. 439 p.

BALDAUF, C.; KUBO, R. R.; SILVA, F.; IRGANG, B. E. "Ferveu, queimou o ser da erva": conhecimentos de especialistas locais sobre plantas medicinais na região Sul do Brasil. Revista Brasileira de Plantas Medicinais, Botucatu, v. 11, n. 3, p. 282-291, 2009.

BENNETT, B. C.; PRANCE, G. T. Introduced plants in the indigenous pharmacopoeia of Northern South America. Economic Botany, v. 54, p. 90-102, 2000.

BRASIL - AGÊNCIA NACIONAL DE VIGILÂNCIA SANITÁRIA. Portaria no 519, de 26 de junho de 1998. Regulamento técnico para fixação de identidade e qualidade de "chás - plantas destinadas à preparação de infusões ou decocções”. 1998. Disponível em: <http://www.anvisa.gov.br/legis/portarias/519_98. htm>. Acesso em: 21 jan. 2012.

BRASIL - CONSELHO NACIONAL DO MEIO AMBIENTE. Resolução CONAMA no 274, de 29 de novembro de 2000. Estabelece a classificação das águas doces, salobras e salinas do Território Nacional. 2000a. Disponível em: <http://www.mma. gov.br/port/conama/res/res274/res27400.html>. Acesso em: 5 mar. 2012.

BRASIL. Portaria Ministério da Saúde no 1.469, de 29 dez. 2000. Estabelece os procedimentos e responsabilidades relativos ao controle e vigilância da qualidade da água para consumo humano e seu padrão de potabilidade, e dá outras providências. $2000 \mathrm{~b}$. Disponível em: <http://www.comitepcj.sp.gov.br/download/ Portaria_MS_1469-00.pdf>. Acesso em: 21 mar. 2012.

BRASIL - AGÊNCIA NACIONAL DE VIGILÂNCIA SANITÁRIA. Resolução RDC 10 de 9 de março de 2010, 10 de março de 2010. Dispõe sobre a notificação de drogas vegetais junto à Agência Nacional de Vigilância Sanitária (ANVISA) e dá outras providências. 2010. Disponível em: <http://www.brasilsus.com.br/ legislacoes/rdc/105008-32.html>. Acesso em: 21 mar. 2010.

BRASILEIRO, B. G.; PIZZIOLO, V. R.; MATOS, D. S.; GERMANO, A. M.; JAMAL, C. M. Plantas medicinais utilizadas pela população atendida no "Programa de Saúde da Família", Governador Valadares, MG, Brasil. Revista Brasileira de Ciências Farmacêuticas, São Paulo, v. 44, p. 4, p. 629-636, 2008.

ELISABETSKY, E.; SETZER, R. Caboclo concepts of disease, diagnosis and therapy: implications for ethnopharmacology and health systems in Amazonia. In: PARKER, E. P. (Ed.). The Amazon caboclo: historical and contemporary perspectives. Williamsburgh: Studies in Third World Societies Publication Series, 1985. p. 243278.

FERRÃO, B. H.; OLIVEIRA, H. B.; MOLINARI, R. F.; TEIXEIRA, M. B.; FONTES, G. G.; AMARO, M. D. O. F. Importância do conhecimento tradicional no uso de plantas medicinais em Buritis, MG, Brasil. Ciência e Natura, Santa Maria, v. 36, ed. especial, p. 321-34, 2014.

MACIEL, M. A. M.; PINTO, A. C.; VEIGA JR., V. F. Plantas medicinais: a necessidade de estudos multidisciplinares. Química Nova, São Paulo, v. 25, n. 3, p. 429-438, 2002.

MARODIN, S. M.; BAPTISTA, L. R. M. O uso de plantas com fins medicinais no município de Dom Pedro de Alcântara, Rio
Grande do Sul, Brasil. Revista Brasileira de Plantas Medicinais, Botucatu, v. 4, n. 1, p. 57-68, 2002.

MARTINS, A. G.; ROSÁRIO, D. L.; BARROS, M. N.; JARDIM, M. A. G. Levantamento etnobotânico de plantas medicinais, alimentares e tóxicas da Ilha do Combu, município de Belém, estado do Pará, Brasil. Revista Brasileira de Farmacognosia, Curitiba, v. 86, n. 1, p. 21-30, 2005.

MARTINS-DA-SILVA, R. C. V. Coleta e identificação de espécimes botânicos. Belém: Embrapa Amazônia Oriental, 2002. $40 \mathrm{p}$.

NEGRELLE, R. R. B.; FORNAZZARI, K. R. C. Estudo etnobotânico em duas comunidades rurais (Limeira e Ribeirão Grande) de Guaratuba (Paraná, Brasil). Revista Brasileira de Plantas Medicinais, Botucatu, v. 9, n. 2, p. 36-54, 2007.

OMS - ORGANIZAÇÃO MUNDIAL DA SAÚDE. Classificação estatística internacional de doenças e problemas relacionados à saúde (CID-10). 8. ed. São Paulo: Editora da Universidade de São Paulo, 2008. 243 p.

PILLA, M. A. C.; AMOROZO, M. C. M.; FURLAN, A. Obtenção e uso das plantas medicinais no distrito de Martim Francisco, município de Mogi-Mirim, SP, Brasil. Acta Botanica Brasilica, Feira de Santana, v. 20, n. 4, p. 789-802, 2006.

PINTO, L. N.; BARBOSA, W. L. R. Etnofarmácia do município de Igarapé-Miri - PA. In: BARBOSA, W. L. R. (Org.). Etnofarmácia: fitoterapia popular e ciência farmacêutica. Belém: NUMA/UFPA, 2009. p. 49-138.

PORRO, A. O Povo das águas: ensaios de etno-história amazônica. Petrópolis: VOZES/EDUSP, 1995. 204 p.

ROHMER, F. O livro do chá. São Paulo: Aquariana, 2002. 256 p. SILVA, A. V.; ALBUQUERQUE, U. P.; NASCIMENTO, V. T. Técnicas para análise de dados etnobiológicos. In: ALBUQUERQUE, U. P.; LUCENA, R. F. P.; CUNHA, L. V. F. C. (Org.). Métodos e técnicas na pesquisa etnobiológica e etnoecológica. Recife: NUPPEA, 2010. p. 187-206.

SILVA, C. S. P.; PROENÇA, C. E. B. Uso e disponibilidade de recursos medicinais no município de Ouro Verde de Goiás, GO, Brasil. Acta Botanica Brasilica, Feira de Santana, v. 22, n. 2, p. 481-492, 2008.

SILVA, J. A.; BÜNDCHEN, M. Conhecimento etnobotânico sobre as plantas medicinais utilizadas pela comunidade do Bairro Cidade Alta, município de Videira, Santa Catarina, Brasil. Unoesc \& Ciência, Joaçaba, v. 2, n. 2, p. 129-140, 2011.

SILVA, N. B.; DEL FINO, A.; ESQUIBEL, M.; SANTOS, J.; ALMEIDA, M. Uso de plantas medicinais na comunidade quilombola da Barra II - Bahia, Brasil. Boletín Latinoamericano y del Caribe de Plantas Medicinales y Aromáticas, Santiago, v. 11, n. 5, p. 435-453, 2012.

SIMÕES, C. M. O.; MENTZ, L. A.; SCHENKEL, E. P.; IRGANG, B. E.; STEHMANN, J. R. Plantas da medicina popular no Rio Grande do Sul. 4 ed. Porto Alegre: Ed. UFRGS, 1995. 173 p.

THRING, T. S. A.; WEITZ, F. M. Medicinal plant use in the bredasdorp/Elim region of the Southern Overberg in the Western Cape Province of South Africa. Journal of Ethnopharmacology, Shannon, v. 103, n. 2, p. 261-275, 2006.

TREVISANATO, S. I.; KIM, Y. I. Tea and health. Nutrition Reviews, Malden, v. 58, n. 1, p. 1-10, 2000. 
TROTTER, R. T.; LOGAN, M. H. Informant consensus: a new approach for identifying potentially effective medicinal plants. In: ETKIN, N. L. (Ed.). Plants in indigenous medicine and diet: biobehavioral approachs. New York: Redgrave Publishing C, 1986. p. 91-112.
VENDRUSCOLO, G. S.; MENTZ, L. A. Estudo da concordância das citações de uso e importância das espécies e famílias utilizadas como medicinais pela comunidade do bairro Ponta Grossa, Porto Alegre, RS, Brasil. Acta Botanica Brasilica, Manaus, v. 20, n. 2, p. 367-382, 2006. 